\title{
The Jordan-Hölder Theorem
}

\author{
Marco Riccardi \\ Casella Postale 49 \\ 54038 Montignoso, Italy
}

\begin{abstract}
Summary. The goal of this article is to formalize the Jordan-Hölder theorem in the context of group with operators as in the book [5]. Accordingly, the article introduces the structure of group with operators and reformulates some theorems on a group already present in the Mizar Mathematical Library. Next, the article formalizes the Zassenhaus butterfly lemma and the Schreier refinement theorem, and defines the composition series.
\end{abstract}

MML identifier: GROUP_9, version: 7.8.05 4.84.971

The terminology and notation used here are introduced in the following articles: [17], [25], [3], [26], [7], [27], [8], [9], [4], [10], [1], [12], [18], [2], [6], [21], [20], [22], [19], [15], [23], [11], [14], [16], [13], and [24].

\section{Actions and Groups with Operators}

Let $O, E$ be sets. An action of $O$ on $E$ is a function from $O$ into $E^{E}$.

Let $O, E$ be sets, let $A$ be an action of $O$ on $E$, and let $I_{1}$ be a set. We say that $I_{1}$ is stable under the action of $A$ if and only if:

(Def. 1) For every element $o$ of $O$ and for every function $f$ from $E$ into $E$ such that $o \in O$ and $f=A(o)$ holds $f^{\circ} I_{1} \subseteq I_{1}$.

Let $O, E$ be sets, let $A$ be an action of $O$ on $E$, and let $X$ be a subset of $E$. The stable subset generated by $X$ yields a subset of $E$ and is defined by the conditions (Def. 2).

(Def. 2)(i) $X \subseteq$ the stable subset generated by $X$,

(ii) the stable subset generated by $X$ is stable under the action of $A$, and

(iii) for every subset $Y$ of $E$ such that $Y$ is stable under the action of $A$ and $X \subseteq Y$ holds the stable subset generated by $X \subseteq Y$. 
Let $O, E$ be sets, let $A$ be an action of $O$ on $E$, and let $F$ be a finite sequence of elements of $O$. The functor $\operatorname{Product}(F, A)$ yields a function from $E$ into $E$ and is defined by:

(Def. 3)(i) $\operatorname{Product}(F, A)=\mathrm{id}_{E}$ if len $F=0$,

(ii) there exists a finite sequence $P_{1}$ of elements of $E^{E}$ such that $\operatorname{Product}(F, A)=P_{1}($ len $F)$ and len $P_{1}=$ len $F$ and $P_{1}(1)=A(F(1))$ and for every natural number $n$ such that $n \neq 0$ and $n<\operatorname{len} F$ there exist functions $f, g$ from $E$ into $E$ such that $f=P_{1}(n)$ and $g=A(F(n+1))$ and $P_{1}(n+1)=f \cdot g$, otherwise.

Let $O$ be a set, let $G$ be a group, and let $I_{1}$ be an action of $O$ on the carrier of $G$. We say that $I_{1}$ is distributive if and only if:

(Def. 4) For every element $o$ of $O$ such that $o \in O$ holds $I_{1}(o)$ is a homomorphism from $G$ to $G$.

Let $O$ be a set. We consider group structures with operators in $O$ as extensions of groupoid as systems

$\langle$ a carrier, a multiplication, an action $\rangle$,

where the carrier is a set, the multiplication is a binary operation on the carrier, and the action is an action of $O$ on the carrier.

Let $O$ be a set. Observe that there exists a group structure with operators in $O$ which is non empty.

Let $O$ be a set and let $I_{1}$ be a non empty group structure with operators in $O$. We say that $I_{1}$ is distributive if and only if the condition (Def. 5) is satisfied.

(Def. 5) Let $G$ be a group and $a$ be an action of $O$ on the carrier of $G$. Suppose $a=$ the action of $I_{1}$ and the groupoid of $G=$ the groupoid of $I_{1}$. Then $a$ is distributive.

Let $O$ be a set. Observe that there exists a non empty group structure with operators in $O$ which is strict, distributive, group-like, and associative.

Let $O$ be a set. A group with operators in $O$ is a distributive group-like associative non empty group structure with operators in $O$.

Let $O$ be a set, let $G$ be a group with operators in $O$, and let $o$ be an element of $O$. The functor $G^{\frown} o$ yields a homomorphism from $G$ to $G$ and is defined as follows:

(Def. 6) $G^{\frown} o=\left\{\begin{array}{l}(\text { the action of } G)(o), \text { if } o \in O, \\ \operatorname{id}_{\text {the carrier of } G, \text { otherwise. }}\end{array}\right.$

Let $O$ be a set and let $G$ be a group with operators in $O$. A distributive group-like associative non empty group structure with operators in $O$ is said to be a stable subgroup of $G$ if:

(Def. 7) It is a subgroup of $G$ and for every element $o$ of $O$ holds it ${ }^{\frown} o=\left(G^{\wedge} o\right)$ 「the carrier of it.

Let $O$ be a set and let $G$ be a group with operators in $O$. Note that there exists a stable subgroup of $G$ which is strict. 
Let $O$ be a set and let $G$ be a group with operators in $O$. The functor $\{\mathbf{1}\}_{G}$ yields a strict stable subgroup of $G$ and is defined by:

(Def. 8) The carrier of $\{\mathbf{1}\}_{G}=\left\{\mathbf{1}_{G}\right\}$.

Let $O$ be a set and let $G$ be a group with operators in $O$. The functor $\Omega_{G}$ yielding a strict stable subgroup of $G$ is defined as follows:

(Def. 9) $\Omega_{G}=$ the group structure with operators of $G$.

Let $O$ be a set, let $G$ be a group with operators in $O$, and let $I_{1}$ be a stable subgroup of $G$. We say that $I_{1}$ is normal if and only if:

(Def. 10) For every strict subgroup $H$ of $G$ such that $H=$ the groupoid of $I_{1}$ holds $H$ is normal.

Let $O$ be a set and let $G$ be a group with operators in $O$. Note that there exists a stable subgroup of $G$ which is strict and normal.

Let $O$ be a set, let $G$ be a group with operators in $O$, and let $H$ be a stable subgroup of $G$. Observe that there exists a stable subgroup of $H$ which is normal.

Let $O$ be a set and let $G$ be a group with operators in $O$. Note that $\{\mathbf{1}\}_{G}$ is normal and $\Omega_{G}$ is normal.

Let $O$ be a set and let $G$ be a group with operators in $O$. The stable subgroups of $G$ yields a set and is defined as follows:

(Def. 11) For every set $x$ holds $x \in$ the stable subgroups of $G$ iff $x$ is a strict stable subgroup of $G$.

Let $O$ be a set and let $G$ be a group with operators in $O$. Observe that the stable subgroups of $G$ is non empty.

Let $I_{1}$ be a group. We say that $I_{1}$ is simple if and only if:

(Def. 12) $I_{1}$ is not trivial and it is not true that there exists a strict normal subgroup $H$ of $I_{1}$ such that $H \neq \Omega_{\left(I_{1}\right)}$ and $H \neq\{\mathbf{1}\}_{\left(I_{1}\right)}$.

Let us note that there exists a group which is strict and simple.

Let $O$ be a set and let $I_{1}$ be a group with operators in $O$. We say that $I_{1}$ is simple if and only if:

(Def. 13) $I_{1}$ is not trivial and it is not true that there exists a strict normal stable subgroup $H$ of $I_{1}$ such that $H \neq \Omega_{\left(I_{1}\right)}$ and $H \neq\{\mathbf{1}\}_{\left(I_{1}\right)}$.

Let $O$ be a set. Observe that there exists a group with operators in $O$ which is strict and simple.

Let $O$ be a set, let $G$ be a group with operators in $O$, and let $N$ be a normal stable subgroup of $G$. The functor Cosets $N$ yields a set and is defined by:

(Def. 14) For every strict normal subgroup $H$ of $G$ such that $H=$ the groupoid of $N$ holds Cosets $N=$ Cosets $H$.

Let $O$ be a set, let $G$ be a group with operators in $O$, and let $N$ be a normal stable subgroup of $G$. The functor $\operatorname{CosOp} N$ yielding a binary operation on Cosets $N$ is defined by: 
(Def. 15) For every strict normal subgroup $H$ of $G$ such that $H=$ the groupoid of $N$ holds $\operatorname{Cos} O p=\operatorname{CosOp} H$.

Let $O$ be a set, let $G$ be a group with operators in $O$, and let $N$ be a normal stable subgroup of $G$. The functor $\operatorname{CosAc} N$ yielding an action of $O$ on Cosets $N$ is defined as follows:

(Def. 16)(i) For every element $o$ of $O$ holds $(\operatorname{CosAc} N)(o)=\{\langle A, B\rangle ; A$ ranges over elements of Cosets $N, B$ ranges over elements of Cosets $N$ : $\left.\bigvee_{g, h \text { : element of } G}\left(g \in A \wedge h \in B \wedge h=\left(G^{\frown} o\right)(g)\right)\right\}$ if $O$ is not empty,

(ii) $\operatorname{CosAc} N=\left[\emptyset,\left\{\operatorname{id}_{\operatorname{Cosets} N}\right\}:\right.$, otherwise.

Let $O$ be a set, let $G$ be a group with operators in $O$, and let $N$ be a normal stable subgroup of $G$. The functor $G / N$ yields a group structure with operators in $O$ and is defined as follows:

(Def. 17) $G / N=\langle\operatorname{Cosets} N, \operatorname{Cos} O p N, \operatorname{CosAc} N\rangle$.

Let $O$ be a set, let $G$ be a group with operators in $O$, and let $N$ be a normal stable subgroup of $G$. Note that $G /{ }_{N}$ is non empty and $G /{ }_{N}$ is distributive, group-like, and associative.

Let $O$ be a set, let $G, H$ be groups with operators in $O$, and let $f$ be a function from $G$ into $H$. We say that $f$ is homomorphic if and only if:

(Def. 18) For every element $o$ of $O$ and for every element $g$ of $G$ holds $f\left(\left(G^{\frown}\right.\right.$ $o)(g))=(H \frown o)(f(g))$.

Let $O$ be a set and let $G, H$ be groups with operators in $O$. One can check that there exists a function from $G$ into $H$ which is multiplicative and homomorphic.

Let $O$ be a set and let $G, H$ be groups with operators in $O$. A homomorphism from $G$ to $H$ is a multiplicative homomorphic function from $G$ into $H$.

Let $O$ be a set, let $G, H, I$ be groups with operators in $O$, let $h$ be a homomorphism from $G$ to $H$, and let $h_{1}$ be a homomorphism from $H$ to $I$. Then $h_{1} \cdot h$ is a homomorphism from $G$ to $I$.

Let $O$ be a set, let $G, H$ be groups with operators in $O$, and let $h$ be a homomorphism from $G$ to $H$. We say that $h$ is monomorphism if and only if:

(Def. 19) $h$ is one-to-one.

We say that $h$ is epimorphism if and only if:

(Def. 20) $\operatorname{rng} h=$ the carrier of $H$.

Let $O$ be a set, let $G, H$ be groups with operators in $O$, and let $h$ be a homomorphism from $G$ to $H$. We say that $h$ is isomorphism if and only if:

(Def. 21) $h$ is an epimorphism and a monomorphism.

Let $O$ be a set and let $G, H$ be groups with operators in $O$. We say that $G$ and $H$ are isomorphic if and only if:

(Def. 22) There exists a homomorphism from $G$ to $H$ which is an isomorphism. 
Let us note that the predicate $G$ and $H$ are isomorphic is reflexive.

Let $O$ be a set and let $G, H$ be groups with operators in $O$. Let us note that the predicate $G$ and $H$ are isomorphic is symmetric.

Let $O$ be a set, let $G$ be a group with operators in $O$, and let $N$ be a normal stable subgroup of $G$. The canonical homomorphism onto cosets of $N$ yields a homomorphism from $G$ to $G / N$ and is defined by the condition (Def. 23).

(Def. 23) Let $H$ be a strict normal subgroup of $G$. Suppose $H=$ the groupoid of $N$. Then the canonical homomorphism onto cosets of $N=$ the canonical homomorphism onto cosets of $H$.

Let $O$ be a set, let $G, H$ be groups with operators in $O$, and let $g$ be a homomorphism from $G$ to $H$. The functor Ker $g$ yields a strict stable subgroup of $G$ and is defined as follows:

(Def. 24) The carrier of Ker $g=\left\{a ; a\right.$ ranges over elements of $\left.G: g(a)=\mathbf{1}_{H}\right\}$.

Let $O$ be a set, let $G, H$ be groups with operators in $O$, and let $g$ be a homomorphism from $G$ to $H$. Observe that $\operatorname{Ker} g$ is normal.

Let $O$ be a set, let $G, H$ be groups with operators in $O$, and let $g$ be a homomorphism from $G$ to $H$. The functor $\operatorname{Im} g$ yielding a strict stable subgroup of $H$ is defined by:

(Def. 25) The carrier of $\operatorname{Im} g=g^{\circ}$ (the carrier of $G$ ).

Let $O$ be a set, let $G$ be a group with operators in $O$, and let $H$ be a stable subgroup of $G$. The functor $\bar{H}$ yielding a subset of $G$ is defined as follows:

(Def. 26) $\bar{H}=$ the carrier of $H$.

Let $O$ be a set, let $G$ be a group with operators in $O$, and let $H_{1}, H_{2}$ be stable subgroups of $G$. The functor $H_{1} \cdot H_{2}$ yields a subset of $G$ and is defined as follows:

(Def. 27) $H_{1} \cdot H_{2}=\overline{H_{1}} \cdot \overline{H_{2}}$.

Let $O$ be a set, let $G$ be a group with operators in $O$, and let $H_{1}, H_{2}$ be stable subgroups of $G$. The functor $H_{1} \cap H_{2}$ yielding a strict stable subgroup of $G$ is defined by:

(Def. 28) The carrier of $H_{1} \cap H_{2}=\overline{H_{1}} \cap \overline{H_{2}}$.

Let us note that the functor $H_{1} \cap H_{2}$ is commutative.

Let $O$ be a set, let $G$ be a group with operators in $O$, and let $A$ be a subset of $G$. The stable subgroup of $A$ yielding a strict stable subgroup of $G$ is defined by the conditions (Def. 29).

(Def. 29)(i) $A \subseteq$ the carrier of the stable subgroup of $A$, and

(ii) for every strict stable subgroup $H$ of $G$ such that $A \subseteq$ the carrier of $H$ holds the stable subgroup of $A$ is a stable subgroup of $H$.

Let $O$ be a set, let $G$ be a group with operators in $O$, and let $H_{1}, H_{2}$ be stable subgroups of $G$. The functor $H_{1} \sqcup H_{2}$ yielding a strict stable subgroup of $G$ is defined as follows: 
(Def. 30) $H_{1} \sqcup H_{2}=$ the stable subgroup of $\overline{H_{1}} \cup \overline{H_{2}}$.

\section{Some Theorems on Groups Reformulated for Groups with OPERATORS}

For simplicity, we follow the rules: $x, O$ are sets, $o$ is an element of $O, G, H$, $I$ are groups with operators in $O, A, B$ are subsets of $G, N$ is a normal stable subgroup of $G, H_{1}, H_{2}, H_{3}$ are stable subgroups of $G, g_{1}, g_{2}$ are elements of $G$, $h_{1}, h_{2}$ are elements of $H_{1}$, and $h$ is a homomorphism from $G$ to $H$.

One can prove the following propositions:

(1) If $x \in H_{1}$, then $x \in G$.

(2) $h_{1}$ is an element of $G$.

(3) If $h_{1}=g_{1}$ and $h_{2}=g_{2}$, then $h_{1} \cdot h_{2}=g_{1} \cdot g_{2}$.

(4) $\mathbf{1}_{G}=\mathbf{1}_{\left(H_{1}\right)}$.

(5) $\mathbf{1}_{G} \in H_{1}$.

(6) If $h_{1}=g_{1}$, then $h_{1}^{-1}=g_{1}{ }^{-1}$.

(7) If $g_{1} \in H_{1}$ and $g_{2} \in H_{1}$, then $g_{1} \cdot g_{2} \in H_{1}$.

(8) If $g_{1} \in H_{1}$, then $g_{1}{ }^{-1} \in H_{1}$.

(9) Suppose that

(i) $A \neq \emptyset$,

(ii) for all $g_{1}, g_{2}$ such that $g_{1} \in A$ and $g_{2} \in A$ holds $g_{1} \cdot g_{2} \in A$,

(iii) for every $g_{1}$ such that $g_{1} \in A$ holds $g_{1}{ }^{-1} \in A$, and

(iv) for all $o, g_{1}$ such that $g_{1} \in A$ holds $\left(G^{\frown} o\right)\left(g_{1}\right) \in A$.

Then there exists a strict stable subgroup $H$ of $G$ such that the carrier of $H=A$.

(10) $G$ is a stable subgroup of $G$.

(11) Let $G_{1}, G_{2}, G_{3}$ be groups with operators in $O$. Suppose $G_{1}$ is a stable subgroup of $G_{2}$ and $G_{2}$ is a stable subgroup of $G_{3}$. Then $G_{1}$ is a stable subgroup of $G_{3}$.

(12) If the carrier of $H_{1} \subseteq$ the carrier of $H_{2}$, then $H_{1}$ is a stable subgroup of $\mathrm{H}_{2}$.

(13) If for every element $g$ of $G$ such that $g \in H_{1}$ holds $g \in H_{2}$, then $H_{1}$ is a stable subgroup of $\mathrm{H}_{2}$.

(14) For all strict stable subgroups $H_{1}, H_{2}$ of $G$ such that the carrier of $H_{1}=$ the carrier of $H_{2}$ holds $H_{1}=H_{2}$.

(15) $\{\mathbf{1}\}_{G}=\{\mathbf{1}\}_{\left(H_{1}\right)}$.

(16) $\{\mathbf{1}\}_{G}$ is a stable subgroup of $H_{1}$.

(17) If $\overline{H_{1}} \cdot \overline{H_{2}}=\overline{H_{2}} \cdot \overline{H_{1}}$, then there exists a strict stable subgroup $H$ of $G$ such that the carrier of $H=\overline{H_{1}} \cdot \overline{H_{2}}$. 
(18)(i) For every stable subgroup $H$ of $G$ such that $H=H_{1} \cap H_{2}$ holds the carrier of $H=$ (the carrier of $\left.H_{1}\right) \cap$ (the carrier of $H_{2}$ ), and

(ii) for every strict stable subgroup $H$ of $G$ such that the carrier of $H=$ (the carrier of $\left.H_{1}\right) \cap$ (the carrier of $H_{2}$ ) holds $H=H_{1} \cap H_{2}$.

(19) For every strict stable subgroup $H$ of $G$ holds $H \cap H=H$.

(20) $\left(H_{1} \cap H_{2}\right) \cap H_{3}=H_{1} \cap\left(H_{2} \cap H_{3}\right)$.

(21) $\{\mathbf{1}\}_{G} \cap H_{1}=\{\mathbf{1}\}_{G}$ and $H_{1} \cap\{\mathbf{1}\}_{G}=\{\mathbf{1}\}_{G}$.

(22) $\bigcup \operatorname{Cosets} N=$ the carrier of $G$.

(23) Let $N_{1}, N_{2}$ be strict normal stable subgroups of $G$. Then there exists a strict normal stable subgroup $N$ of $G$ such that the carrier of $N=\overline{N_{1}} \cdot \overline{N_{2}}$.

(24) $g_{1} \in$ the stable subgroup of $A$ if and only if there exists a finite sequence $F$ of elements of the carrier of $G$ and there exists a finite sequence $I$ of elements of $\mathbb{Z}$ and there exists a subset $C$ of $G$ such that $C=$ the stable subset generated by $A$ and len $F=\operatorname{len} I$ and $\operatorname{rng} F \subseteq C$ and $\prod\left(F^{I}\right)=g_{1}$.

(25) For every strict stable subgroup $H$ of $G$ holds the stable subgroup of $\bar{H}=H$.

(26) If $A \subseteq B$, then the stable subgroup of $A$ is a stable subgroup of the stable subgroup of $B$.

The scheme MeetSbgWOpEx deals with a set $\mathcal{A}$, a group $\mathcal{B}$ with operators in $\mathcal{A}$, and a unary predicate $\mathcal{P}$, and states that:

There exists a strict stable subgroup $H$ of $\mathcal{B}$ such that the carrier of $H=\bigcap\{A ; A$ ranges over subsets of $\mathcal{B}$ : $\bigvee_{K}$ : strict stable subgroup of $\mathcal{B}(A=$ the carrier of $\left.K \wedge \mathcal{P}[K])\right\}$

provided the parameters meet the following requirement:

- There exists a strict stable subgroup $H$ of $\mathcal{B}$ such that $\mathcal{P}[H]$.

The following propositions are true:

(27) The carrier of the stable subgroup of $A=\bigcap\{B ; B$ ranges over subsets of $G: \bigvee_{H}$ : strict stable subgroup of $G(B=$ the carrier of $\left.H \wedge A \subseteq \bar{H})\right\}$.

(28) For all strict normal stable subgroups $N_{1}, N_{2}$ of $G$ holds $N_{1} \cdot N_{2}=N_{2} \cdot N_{1}$.

(29) $H_{1} \sqcup H_{2}=$ the stable subgroup of $H_{1} \cdot H_{2}$.

(30) If $H_{1} \cdot H_{2}=H_{2} \cdot H_{1}$, then the carrier of $H_{1} \sqcup H_{2}=H_{1} \cdot H_{2}$.

(31) For all strict normal stable subgroups $N_{1}, N_{2}$ of $G$ holds the carrier of $N_{1} \sqcup N_{2}=N_{1} \cdot N_{2}$.

(32) For all strict normal stable subgroups $N_{1}, N_{2}$ of $G$ holds $N_{1} \sqcup N_{2}$ is a normal stable subgroup of $G$.

(33) For every strict stable subgroup $H$ of $G$ holds $\{\mathbf{1}\}_{G} \sqcup H=H$ and $H \sqcup\{\mathbf{1}\}_{G}=H$.

(34) $\Omega_{G} \sqcup H_{1}=\Omega_{G}$ and $H_{1} \sqcup \Omega_{G}=\Omega_{G}$. 
(35) $H_{1}$ is a stable subgroup of $H_{1} \sqcup H_{2}$ and $H_{2}$ is a stable subgroup of $H_{1} \sqcup H_{2}$.

(36) For every strict stable subgroup $H_{2}$ of $G$ holds $H_{1}$ is a stable subgroup of $H_{2}$ iff $H_{1} \sqcup H_{2}=H_{2}$.

(37) Let $H_{3}$ be a strict stable subgroup of $G$. Suppose $H_{1}$ is a stable subgroup of $H_{3}$ and $H_{2}$ is a stable subgroup of $H_{3}$. Then $H_{1} \sqcup H_{2}$ is a stable subgroup of $\mathrm{H}_{3}$.

(38) Let $H_{2}, H_{3}$ be strict stable subgroups of $G$. Suppose $H_{1}$ is a stable subgroup of $H_{2}$. Then $H_{1} \sqcup H_{3}$ is a stable subgroup of $H_{2} \sqcup H_{3}$.

(39) For all stable subgroups $X, Y$ of $H_{1}$ and for all stable subgroups $X^{\prime}, Y^{\prime}$ of $G$ such that $X=X^{\prime}$ and $Y=Y^{\prime}$ holds $X^{\prime} \cap Y^{\prime}=X \cap Y$.

(40) If $N$ is a stable subgroup of $H_{1}$, then $N$ is a normal stable subgroup of $H_{1}$.

(41) $H_{1} \cap N$ is a normal stable subgroup of $H_{1}$ and $N \cap H_{1}$ is a normal stable subgroup of $H_{1}$

(42) For every strict group $G$ with operators in $O$ such that $G$ is trivial holds $\{\mathbf{1}\}_{G}=G$.

(43) $\boldsymbol{1}_{G / N}=\bar{N}$.

(44) Let $M, N$ be strict normal stable subgroups of $G$ and $M_{1}$ be a normal stable subgroup of $N$. Suppose $M_{1}=M$ and $M$ is a stable subgroup of $N$. Then ${ }^{N} / M_{1}$ is a normal stable subgroup of $G / M$.

(45) $h\left(\mathbf{1}_{G}\right)=\mathbf{1}_{H}$.

(46) $h\left(g_{1}^{-1}\right)=h\left(g_{1}\right)^{-1}$.

(47) $g_{1} \in \operatorname{Ker} h$ iff $h\left(g_{1}\right)=\mathbf{1}_{H}$.

(48) For every strict normal stable subgroup $N$ of $G$ holds Ker (the canonical homomorphism onto cosets of $N)=N$.

(49) $\operatorname{rng} h=$ the carrier of $\operatorname{Im} h$.

(50) $\operatorname{Im}$ (the canonical homomorphism onto cosets of $N)={ }^{G} / N$.

(51) Let $H$ be a strict group with operators in $O$ and $h$ be a homomorphism from $G$ to $H$. Then $h$ is an epimorphism if and only if $\operatorname{Im} h=H$.

(52) Let $H$ be a strict group with operators in $O$ and $h$ be a homomorphism from $G$ to $H$. Suppose $h$ is an epimorphism. Let $c$ be an element of $H$. Then there exists an element $a$ of $G$ such that $h(a)=c$.

(53) The canonical homomorphism onto cosets of $N$ is an epimorphism.

(54) The canonical homomorphism onto cosets of $\{\mathbf{1}\}_{G}$ is an isomorphism.

(55) If $G$ and $H$ are isomorphic and $H$ and $I$ are isomorphic, then $G$ and $I$ are isomorphic.

(56) For every strict group $G$ with operators in $O$ holds $G$ and $G /\{\mathbf{1}\}_{G}$ are isomorphic. 
(57) For every strict group $G$ with operators in $O$ holds $G / \Omega_{G}$ is trivial.

(58) Let $G, H$ be strict groups with operators in $O$. If $G$ and $H$ are isomorphic and $G$ is trivial, then $H$ is trivial.

(59) $G / \operatorname{Ker} h$ and $\operatorname{Im} h$ are isomorphic.

(60) Let $H, F_{1}, F_{2}$ be strict stable subgroups of $G$. Suppose $F_{1}$ is a normal stable subgroup of $F_{2}$. Then $H \cap F_{1}$ is a normal stable subgroup of $H \cap F_{2}$.

\section{Others Theorems on Actions and Groups with Operators}

In the sequel $E$ is a set, $A$ is an action of $O$ on $E, C$ is a subset of $G$, and $N_{1}$ is a normal stable subgroup of $H_{1}$.

One can prove the following propositions:

(61) $\Omega_{E}$ is stable under the action of $A$.

(62) $\quad: O,\left\{\operatorname{id}_{E}\right\}:$ is an action of $O$ on $E$.

(63) Let $O$ be a non empty set, $E$ be a set, $o$ be an element of $O$, and $A$ be an action of $O$ on $E$. Then $\operatorname{Product}(\langle o\rangle, A)=A(o)$.

(64) Let $O$ be a non empty set, $E$ be a set, $F_{1}, F_{2}$ be finite sequences of elements of $O$, and $A$ be an action of $O$ on $E$. Then $\operatorname{Product}\left(F_{1} \frown F_{2}, A\right)=$ $\operatorname{Product}\left(F_{1}, A\right) \cdot \operatorname{Product}\left(F_{2}, A\right)$.

(65) Let $F$ be a finite sequence of elements of $O$ and $Y$ be a subset of $E$. If $Y$ is stable under the action of $A$, then $(\operatorname{Product}(F, A))^{\circ} Y \subseteq Y$.

(66) Let $E$ be a non empty set, $A$ be an action of $O$ on $E, X$ be a subset of $E$, and $a$ be an element of $E$. Suppose $X$ is not empty. Then $a \in$ the stable subset generated by $X$ if and only if there exists a finite sequence $F$ of elements of $O$ and there exists an element $x$ of $X$ such that $(\operatorname{Product}(F, A))(x)=a$.

(67) For every strict group $G$ there exists a strict group $H$ with operators in $O$ such that $G=$ the groupoid of $H$.

(68) The groupoid of $H_{1}$ is a strict subgroup of $G$.

(69) The groupoid of $N$ is a strict normal subgroup of $G$.

(70) If $g_{1} \in H_{1}$, then $\left(G^{\frown o} o\right)\left(g_{1}\right) \in H_{1}$.

(71) Let $O$ be a set, $G, H$ be groups with operators in $O, G^{\prime}$ be a strict stable subgroup of $G$, and $f$ be a homomorphism from $G$ to $H$. Then there exists a strict stable subgroup $H^{\prime}$ of $H$ such that the carrier of $H^{\prime}=f^{\circ}\left(\right.$ the carrier of $\left.G^{\prime}\right)$.

(72) If $B$ is empty, then the stable subgroup of $B=\{\mathbf{1}\}_{G}$.

(73) If $B=$ the carrier of $\operatorname{gr}(C)$, then the stable subgroup of $C=$ the stable subgroup of $B$. 
(74) Let $N^{\prime}$ be a normal subgroup of $G$. Suppose $N^{\prime}=$ the groupoid of $N$. Then $G / N^{\prime}=$ the groupoid of $G / N$ and $\mathbf{1}_{G / N^{\prime}}=\mathbf{1}_{G / N}$.

(75) Suppose the carrier of $H_{1}=$ the carrier of $H_{2}$. Then the group structure with operators of $H_{1}=$ the group structure with operators of $\mathrm{H}_{2}$.

(76) Suppose ${ }^{H_{1}} / N_{1}$ is trivial. Then the group structure with operators of $H_{1}=$ the group structure with operators of $N_{1}$.

(77) If the carrier of $H_{1}=$ the carrier of $N_{1}$, then $H_{1} / N_{1}$ is trivial.

(78) Let $G, H$ be groups with operators in $O, N$ be a stable subgroup of $G$, $H^{\prime}$ be a strict stable subgroup of $H$, and $f$ be a homomorphism from $G$ to $H$. Suppose $N=\operatorname{Ker} f$. Then there exists a strict stable subgroup $G^{\prime}$ of $G$ such that

(i) the carrier of $G^{\prime}=f^{-1}$ (the carrier of $H^{\prime}$ ), and

(ii) if $H^{\prime}$ is normal, then $N$ is a normal stable subgroup of $G^{\prime}$ and $G^{\prime}$ is normal.

(79) Let $G, H$ be groups with operators in $O, N$ be a stable subgroup of $G$, $G^{\prime}$ be a strict stable subgroup of $G$, and $f$ be a homomorphism from $G$ to $H$. Suppose $N=\operatorname{Ker} f$. Then there exists a strict stable subgroup $H^{\prime}$ of $H$ such that

(i) the carrier of $H^{\prime}=f^{\circ}$ (the carrier of $G^{\prime}$ ),

(ii) $f^{-1}$ (the carrier of $H^{\prime}$ ) $=$ the carrier of $G^{\prime} \sqcup N$, and

(iii) if $f$ is an epimorphism and $G^{\prime}$ is normal, then $H^{\prime}$ is normal.

(80) Let $G$ be a strict group with operators in $O, N$ be a strict normal stable subgroup of $G$, and $H$ be a strict stable subgroup of $G / N$. Suppose the carrier of $G=(\text { the canonical homomorphism onto cosets of } N)^{-1}$ (the carrier of $H$ ). Then $H=\Omega_{G / N}$.

(81) Let $G$ be a strict group with operators in $O, N$ be a strict normal stable subgroup of $G$, and $H$ be a strict stable subgroup of $G / N$. Suppose the carrier of $N=(\text { the canonical homomorphism onto cosets of } N)^{-1}$ (the carrier of $H$ ). Then $H=\{\mathbf{1}\}_{G / N}$.

(82) Let $G, H$ be strict groups with operators in $O$. If $G$ and $H$ are isomorphic and $G$ is simple, then $H$ is simple.

(83) Let $G$ be a group with operators in $O, H$ be a stable subgroup of $G, F_{3}$ be a finite sequence of elements of the carrier of $G, F_{4}$ be a finite sequence of elements of the carrier of $H$, and $I$ be a finite sequence of elements of $\mathbb{Z}$. If $F_{3}=F_{4}$ and len $F_{3}=$ len $I$, then $\prod\left(F_{3}{ }^{I}\right)=\prod\left(F_{4}{ }^{I}\right)$.

(84) Let $O, E_{1}, E_{2}$ be sets, $A_{1}$ be an action of $O$ on $E_{1}, A_{2}$ be an action of $O$ on $E_{2}$, and $F$ be a finite sequence of elements of $O$. Suppose that

(i) $E_{1} \subseteq E_{2}$, and

(ii) for every element $o$ of $O$ and for every function $f_{1}$ from $E_{1}$ into $E_{1}$ and for every function $f_{2}$ from $E_{2}$ into $E_{2}$ such that $f_{1}=A_{1}(o)$ and $f_{2}=A_{2}(o)$ 
holds $f_{1}=f_{2} \uparrow E_{1}$.

Then $\operatorname{Product}\left(F, A_{1}\right)=\operatorname{Product}\left(F, A_{2}\right)\left\lceil E_{1}\right.$.

(85) Let $N_{1}, N_{2}$ be strict stable subgroups of $H_{1}$ and $N_{1}^{\prime}, N_{2}^{\prime}$ be strict stable subgroups of $G$. If $N_{1}=N_{1}^{\prime}$ and $N_{2}=N_{2}^{\prime}$, then $N_{1}^{\prime} \cdot N_{2}^{\prime}=N_{1} \cdot N_{2}$.

(86) Let $N_{1}, N_{2}$ be strict stable subgroups of $H_{1}$ and $N_{1}^{\prime}, N_{2}^{\prime}$ be strict stable subgroups of $G$. If $N_{1}=N_{1}^{\prime}$ and $N_{2}=N_{2}^{\prime}$, then $N_{1}^{\prime} \sqcup N_{2}^{\prime}=N_{1} \sqcup N_{2}$.

(87) Let $N_{1}, N_{2}$ be strict stable subgroups of $G$. Suppose $N_{1}$ is a normal stable subgroup of $H_{1}$ and $N_{2}$ is a normal stable subgroup of $H_{1}$. Then $N_{1} \sqcup N_{2}$ is a normal stable subgroup of $H_{1}$.

(88) Let $f$ be a homomorphism from $G$ to $H$ and $g$ be a homomorphism from $H$ to $I$. Then the carrier of $\operatorname{Ker}(g \cdot f)=f^{-1}$ (the carrier of $\operatorname{Ker} g$ ).

(89) Let $G^{\prime}$ be a stable subgroup of $G, H^{\prime}$ be a stable subgroup of $H$, and $f$ be a homomorphism from $G$ to $H$. Suppose the carrier of $H^{\prime}=f^{\circ}$ (the carrier of $G^{\prime}$ ) or the carrier of $G^{\prime}=f^{-1}$ (the carrier of $H^{\prime}$ ). Then $f$ the carrier of $G^{\prime}$ is a homomorphism from $G^{\prime}$ to $H^{\prime}$.

(90) Let $G, H$ be strict groups with operators in $O, N, L, G^{\prime}$ be strict stable subgroups of $G$, and $f$ be a homomorphism from $G$ to $H$. Suppose $N=$ Ker $f$ and $L$ is a strict normal stable subgroup of $G^{\prime}$. Then

(i) $L \sqcup G^{\prime} \cap N$ is a normal stable subgroup of $G^{\prime}$,

(ii) $L \sqcup N$ is a normal stable subgroup of $G^{\prime} \sqcup N$, and

(iii) for every strict normal stable subgroup $N_{1}$ of $G^{\prime} \sqcup N$ and for every strict normal stable subgroup $N_{2}$ of $G^{\prime}$ such that $N_{1}=L \sqcup N$ and $N_{2}=L \sqcup G^{\prime} \cap N$ holds $\left(G^{\prime} \sqcup N\right) / N_{1}$ and $G^{\prime} / N_{2}$ are isomorphic.

\section{The Zassenhaus Butterfly Lemma}

The following propositions are true:

(91) Let $H, K, H^{\prime}, K^{\prime}$ be strict stable subgroups of $G, J_{1}$ be a normal stable subgroup of $H^{\prime} \sqcup H \cap K$, and $H_{4}$ be a normal stable subgroup of $H \cap K$. Suppose $H^{\prime}$ is a normal stable subgroup of $H$ and $K^{\prime}$ is a normal stable subgroup of $K$ and $J_{1}=H^{\prime} \sqcup H \cap K^{\prime}$ and $H_{4}=H^{\prime} \cap K \sqcup K^{\prime} \cap H$. Then $\left(H^{\prime} \sqcup H \cap K\right) / J_{1}$ and $(H \cap K) / H_{4}$ are isomorphic.

(92) Let $H, K, H^{\prime}, K^{\prime}$ be strict stable subgroups of $G$. Suppose $H^{\prime}$ is a normal stable subgroup of $H$ and $K^{\prime}$ is a normal stable subgroup of $K$. Then $H^{\prime} \sqcup H \cap K^{\prime}$ is a normal stable subgroup of $H^{\prime} \sqcup H \cap K$.

(93) Let $H, K, H^{\prime}, K^{\prime}$ be strict stable subgroups of $G, J_{1}$ be a normal stable subgroup of $H^{\prime} \sqcup H \cap K$, and $J_{2}$ be a normal stable subgroup of $K^{\prime} \sqcup K \cap H$. Suppose $J_{1}=H^{\prime} \sqcup H \cap K^{\prime}$ and $J_{2}=K^{\prime} \sqcup K \cap H^{\prime}$ and $H^{\prime}$ is a normal stable subgroup of $H$ and $K^{\prime}$ is a normal stable subgroup of $K$. Then $\left(H^{\prime} \sqcup H \cap K\right) / J_{1}$ and $\left(K^{\prime} \sqcup K \cap H\right) / J_{2}$ are isomorphic. 


\section{Composition Series}

Let $O$ be a set, let $G$ be a group with operators in $O$, and let $I_{1}$ be a finite sequence of elements of the stable subgroups of $G$. We say that $I_{1}$ is composition series if and only if the conditions (Def. 31) are satisfied.

(Def. 31)(i) $\quad I_{1}(1)=\Omega_{G}$,

(ii) $I_{1}\left(\right.$ len $\left.I_{1}\right)=\{\mathbf{1}\}_{G}$, and

(iii) for every natural number $i$ such that $i \in \operatorname{dom} I_{1}$ and $i+1 \in \operatorname{dom} I_{1}$ and for all stable subgroups $H_{1}, H_{2}$ of $G$ such that $H_{1}=I_{1}(i)$ and $H_{2}=$ $I_{1}(i+1)$ holds $H_{2}$ is a normal stable subgroup of $H_{1}$.

Let $O$ be a set and let $G$ be a group with operators in $O$. One can verify that there exists a finite sequence of elements of the stable subgroups of $G$ which is composition series.

Let $O$ be a set and let $G$ be a group with operators in $O$. A composition series of $G$ is a composition series finite sequence of elements of the stable subgroups of $G$.

Let $O$ be a set, let $G$ be a group with operators in $O$, and let $s_{1}, s_{2}$ be composition series of $G$. We say that $s_{1}$ is finer than $s_{2}$ if and only if:

(Def. 32) There exists a set $x$ such that $x \subseteq \operatorname{dom} s_{1}$ and $s_{2}=s_{1} \cdot \operatorname{Sgm} x$.

Let us note that the predicate $s_{1}$ is finer than $s_{2}$ is reflexive.

Let $O$ be a set, let $G$ be a group with operators in $O$, and let $I_{1}$ be a composition series of $G$. We say that $I_{1}$ is strictly decreasing if and only if the condition (Def. 33) is satisfied.

(Def. 33) Let $i$ be a natural number. Suppose $i \in \operatorname{dom} I_{1}$ and $i+1 \in \operatorname{dom} I_{1}$. Let $H$ be a stable subgroup of $G$ and $N$ be a normal stable subgroup of $H$. If $H=I_{1}(i)$ and $N=I_{1}(i+1)$, then ${ }^{H} / N$ is not trivial.

Let $O$ be a set, let $G$ be a group with operators in $O$, and let $I_{1}$ be a composition series of $G$. We say that $I_{1}$ is Jordan-Hölder if and only if the conditions (Def. 34) are satisfied.

(Def. 34)(i) $\quad I_{1}$ is strictly decreasing, and

(ii) it is not true that there exists a composition series $s$ of $G$ such that $s \neq I_{1}$ and $s$ is strictly decreasing and finer than $I_{1}$.

Let $O$ be a set, let $G_{1}, G_{2}$ be groups with operators in $O$, let $s_{1}$ be a composition series of $G_{1}$, and let $s_{2}$ be a composition series of $G_{2}$. We say that $s_{1}$ is equivalent with $s_{2}$ if and only if the conditions (Def. 35) are satisfied.

(Def. 35)(i) len $s_{1}=$ len $s_{2}$, and

(ii) for every natural number $n$ such that $n+1=$ len $s_{1}$ there exists a permutation $p$ of $\operatorname{Seg} n$ such that for every stable subgroup $H_{1}$ of $G_{1}$ and for every stable subgroup $H_{2}$ of $G_{2}$ and for every normal stable subgroup $N_{1}$ of $H_{1}$ and for every normal stable subgroup $N_{2}$ of $H_{2}$ and for all natural numbers $i, j$ such that $1 \leq i$ and $i \leq n$ and $j=p(i)$ and $H_{1}=s_{1}(i)$ and 
$H_{2}=s_{2}(j)$ and $N_{1}=s_{1}(i+1)$ and $N_{2}=s_{2}(j+1)$ holds ${ }^{H_{1}} / N_{1}$ and ${ }^{H_{2}} / N_{2}$ are isomorphic.

Let $O$ be a set, let $G$ be a group with operators in $O$, and let $s$ be a composition series of $G$. The series of quotients of $s$ yielding a finite sequence is defined as follows:

(Def. 36)(i) len $s=$ len (the series of quotients of $s)+1$ and for every natural number $i$ such that $i \in \operatorname{dom}$ (the series of quotients of $s$ ) and for every stable subgroup $H$ of $G$ and for every normal stable subgroup $N$ of $H$ such that $H=s(i)$ and $N=s(i+1)$ holds (the series of quotients of $s)(i)={ }^{H} / N$ if len $s>1$,

(ii) the series of quotients of $s=\emptyset$, otherwise.

Let $O$ be a set, let $f_{1}, f_{2}$ be finite sequences, and let $p$ be a permutation of $\operatorname{dom} f_{1}$. We say that $f_{1}$ and $f_{2}$ are equivalent under $p$ in $O$ if and only if the conditions (Def. 37) are satisfied.

(Def. 37)(i) len $f_{1}=\operatorname{len} f_{2}$, and

(ii) for all groups $H_{1}, H_{2}$ with operators in $O$ and for all natural numbers $i, j$ such that $i \in \operatorname{dom} f_{1}$ and $j=p^{-1}(i)$ and $H_{1}=f_{1}(i)$ and $H_{2}=f_{2}(j)$ holds $H_{1}$ and $H_{2}$ are isomorphic.

For simplicity, we follow the rules: $y$ is a set, $s_{1}, s_{1}^{\prime}, s_{2}, s_{2}^{\prime}$ are composition series of $G, f_{3}$ is a finite sequence of elements of the stable subgroups of $G, f_{1}$, $f_{2}$ are finite sequences, and $i, j, n$ are natural numbers.

We now state a number of propositions:

(94) If $i \in \operatorname{dom} s_{1}$ and $i+1 \in \operatorname{dom} s_{1}$ and $s_{1}(i)=s_{1}(i+1)$ and $f_{3}=\left(s_{1}\right)_{\uparrow i}$, then $f_{3}$ is composition series.

(95) If $s_{1}$ is finer than $s_{2}$, then there exists $n$ such that len $s_{1}=$ len $s_{2}+n$.

(96) If len $s_{2}=$ len $s_{1}$ and $s_{2}$ is finer than $s_{1}$, then $s_{1}=s_{2}$.

(97) If $s_{1}$ is not empty and $s_{2}$ is finer than $s_{1}$, then $s_{2}$ is not empty.

(98) If $s_{1}$ is finer than $s_{2}$ and Jordan-Hölder and $s_{2}$ is Jordan-Hölder, then $s_{1}=s_{2}$.

(99) If $i \in \operatorname{dom} s_{1}$ and $i+1 \in \operatorname{dom} s_{1}$ and $s_{1}(i)=s_{1}(i+1)$ and $s_{1}^{\prime}=\left(s_{1}\right)_{\uparrow i}$ and $s_{2}$ is Jordan-Hölder and $s_{1}$ is finer than $s_{2}$, then $s_{1}^{\prime}$ is finer than $s_{2}$.

(100) Suppose len $s_{1}>1$ and $s_{2} \neq s_{1}$ and $s_{2}$ is strictly decreasing and finer than $s_{1}$. Then there exist $i, j$ such that $i \in \operatorname{dom} s_{1}$ and $i \in \operatorname{dom} s_{2}$ and $i+1 \in \operatorname{dom} s_{1}$ and $i+1 \in \operatorname{dom} s_{2}$ and $j \in \operatorname{dom} s_{2}$ and $i+1<j$ and $s_{1}(i)=s_{2}(i)$ and $s_{1}(i+1) \neq s_{2}(i+1)$ and $s_{1}(i+1)=s_{2}(j)$.

(101) If $i \in \operatorname{dom} s_{1}$ and $j \in \operatorname{dom} s_{1}$ and $i \leq j$ and $H_{1}=s_{1}(i)$ and $H_{2}=s_{1}(j)$, then $H_{2}$ is a stable subgroup of $H_{1}$.

(102) If $y \in \operatorname{rng}$ (the series of quotients of $s_{1}$ ), then $y$ is a strict group with operators in $O$. 
(103) Suppose $i \in$ dom (the series of quotients of $s_{1}$ ) and for every $H$ such that $H=\left(\right.$ the series of quotients of $\left.s_{1}\right)(i)$ holds $H$ is trivial. Then $i \in \operatorname{dom} s_{1}$ and $i+1 \in \operatorname{dom} s_{1}$ and $s_{1}(i)=s_{1}(i+1)$.

(104) Suppose $i \in \operatorname{dom} s_{1}$ and $i+1 \in \operatorname{dom} s_{1}$ and $s_{1}(i)=s_{1}(i+1)$ and $s_{2}=\left(s_{1}\right)_{\uparrow i}$. Then the series of quotients of $s_{2}=$ (the series of quotients of $\left.s_{1}\right)_{\uparrow i}$.

(105) Suppose $f_{1}=$ the series of quotients of $s_{1}$ and $i \in \operatorname{dom} f_{1}$ and for every $H$ such that $H=f_{1}(i)$ holds $H$ is trivial. Then $\left(s_{1}\right)_{\uparrow i}$ is a composition series of $G$ and for every $s_{2}$ such that $s_{2}=\left(s_{1}\right)_{\uparrow i}$ holds the series of quotients of $s_{2}=\left(f_{1}\right)_{\uparrow i}$.

(106) Suppose that

(i) $f_{1}=$ the series of quotients of $s_{1}$,

(ii) $f_{2}=$ the series of quotients of $s_{2}$,

(iii) $i \in \operatorname{dom} f_{1}$,

(iv) for every $H$ such that $H=f_{1}(i)$ holds $H$ is trivial, and

(v) there exists a permutation $p$ of $\operatorname{dom} f_{1}$ such that $f_{1}$ and $f_{2}$ are equivalent under $p$ in $O$ and $j=p^{-1}(i)$.

Then there exists a permutation $p^{\prime}$ of $\operatorname{dom}\left(\left(f_{1}\right)_{\uparrow i}\right)$ such that $\left(f_{1}\right)_{\uparrow i}$ and $\left(f_{2}\right)_{\vdash j}$ are equivalent under $p^{\prime}$ in $O$.

(107) Let $G_{1}, G_{2}$ be groups with operators in $O, s_{1}$ be a composition series of $G_{1}$, and $s_{2}$ be a composition series of $G_{2}$. If $s_{1}$ is empty and $s_{2}$ is empty, then $s_{1}$ is equivalent with $s_{2}$.

(108) Let $G_{1}, G_{2}$ be groups with operators in $O, s_{1}$ be a composition series of $G_{1}$, and $s_{2}$ be a composition series of $G_{2}$. Suppose $s_{1}$ is not empty and $s_{2}$ is not empty. Then $s_{1}$ is equivalent with $s_{2}$ if and only if there exists a permutation $p$ of dom (the series of quotients of $s_{1}$ ) such that the series of quotients of $s_{1}$ and the series of quotients of $s_{2}$ are equivalent under $p$ in $O$.

(109) Suppose $s_{1}$ is finer than $s_{2}$ and $s_{2}$ is Jordan-Hölder and len $s_{1}>$ len $s_{2}$. Then there exists $i$ such that $i \in \operatorname{dom}$ (the series of quotients of $s_{1}$ ) and for every $H$ such that $H=$ (the series of quotients of $\left.s_{1}\right)(i)$ holds $H$ is trivial.

(110) Suppose len $s_{1}>1$. Then $s_{1}$ is Jordan-Hölder if and only if for every $i$ such that $i \in \operatorname{dom}$ (the series of quotients of $s_{1}$ ) holds (the series of quotients of $\left.s_{1}\right)(i)$ is a strict simple group with operators in $O$.

(111) Suppose $1 \leq i$ and $i \leq$ len $s_{1}-1$. Then $s_{1}(i)$ is a strict stable subgroup of $G$ and $s_{1}(i+1)$ is a strict stable subgroup of $G$.

(112) If $1 \leq i$ and $i \leq$ len $s_{1}-1$ and $H_{1}=s_{1}(i)$ and $H_{2}=s_{1}(i+1)$, then $H_{2}$ is a normal stable subgroup of $H_{1}$.

(113) $s_{1}$ is equivalent with $s_{1}$. 
(114) If len $s_{1} \leq 1$ or len $s_{2} \leq 1$ and if len $s_{1} \leq$ len $s_{2}$, then $s_{2}$ is finer than $s_{1}$.

(115) If $s_{1}$ is equivalent with $s_{2}$ and Jordan-Hölder, then $s_{2}$ is Jordan-Hölder.

\section{The Schreier Refinement Theorem}

Let us consider $O, G, s_{1}, s_{2}$. Let us assume that len $s_{1}>1$ and len $s_{2}>1$. The Schreier series of $s_{1}$ and $s_{2}$ yielding a composition series of $G$ is defined by the condition (Def. 38).

(Def. 38) Let $k, i, j$ be natural numbers and $H_{1}, H_{2}, H_{3}$ be stable subgroups of $G$. Then

(i) $\quad$ if $k=(i-1) \cdot\left(\right.$ len $\left.s_{2}-1\right)+j$ and $1 \leq i$ and $i \leq$ len $s_{1}-1$ and $1 \leq j$ and $j \leq$ len $s_{2}-1$ and $H_{1}=s_{1}(i+1)$ and $H_{2}=s_{1}(i)$ and $H_{3}=s_{2}(j)$, then (the Schreier series of $s_{1}$ and $\left.s_{2}\right)(k)=H_{1} \sqcup H_{2} \cap H_{3}$,

(ii) if $k=\left(\right.$ len $\left.s_{1}-1\right) \cdot\left(\right.$ len $\left.s_{2}-1\right)+1$, then (the Schreier series of $s_{1}$ and $\left.s_{2}\right)(k)=\{\mathbf{1}\}_{G}$, and

(iii) $\quad$ len (the Schreier series of $s_{1}$ and $\left.s_{2}\right)=\left(\right.$ len $\left.s_{1}-1\right) \cdot\left(\right.$ len $\left.s_{2}-1\right)+1$.

Next we state three propositions:

(116) If len $s_{1}>1$ and len $s_{2}>1$, then the Schreier series of $s_{1}$ and $s_{2}$ is finer than $s_{1}$.

(117) If len $s_{1}>1$ and len $s_{2}>1$, then the Schreier series of $s_{1}$ and $s_{2}$ is equivalent with the Schreier series of $s_{2}$ and $s_{1}$.

(118) There exist $s_{1}^{\prime}, s_{2}^{\prime}$ such that $s_{1}^{\prime}$ is finer than $s_{1}$ and $s_{2}^{\prime}$ is finer than $s_{2}$ and $s_{1}^{\prime}$ is equivalent with $s_{2}^{\prime}$.

\section{The Jordan-HÖLder TheOREM}

One can prove the following proposition

(119) If $s_{1}$ is Jordan-Hölder and $s_{2}$ is Jordan-Hölder, then $s_{1}$ is equivalent with $s_{2}$.

\section{ApPEndix}

Next we state several propositions:

(120) For all binary relations $P, R$ holds $P=\operatorname{rng} P\left\lceil R\right.$ iff $P^{\smile}=R^{\smile} \uparrow \operatorname{dom}\left(P^{\smile}\right)$.

(121) For every set $X$ and for all binary relations $P, R$ holds $P \cdot(R \uparrow X)=$ $(X\lceil P) \cdot R$

(122) Let $n$ be a natural number, $X$ be a set, and $f$ be a partial function from $\mathbb{R}$ to $\mathbb{R}$. If $X \subseteq \operatorname{Seg} n$ and $X \subseteq \operatorname{dom} f$ and $f$ is increasing on $X$ and $f^{\circ} X \subseteq \mathbb{N} \backslash\{0\}$, then $\operatorname{Sgm}\left(f^{\circ} X\right)=f \cdot \operatorname{Sgm} X$. 
(123) Let $y$ be a set and $i, n$ be natural numbers. Suppose $y \subseteq \operatorname{Seg}(n+1)$ and $i \in \operatorname{Seg}(n+1)$ and $i \notin y$. Then there exists $x$ such that $\operatorname{Sgm} x=$ $(\operatorname{Sgm}(\operatorname{Seg}(n+1) \backslash\{i\}))^{-1} \cdot \operatorname{Sgm} y$ and $x \subseteq \operatorname{Seg} n$.

(124) Let $D$ be a non empty set, $f$ be a finite sequence of elements of $D$, $p$ be an element of $D$, and $n$ be an element of $\mathbb{N}$. If $n \in \operatorname{dom} f$, then $f=(\operatorname{Ins}(f, n, p))_{\lceil n+1}$.

(125) Let $G, H$ be groups, $F_{1}$ be a finite sequence of elements of the carrier of $G, F_{2}$ be a finite sequence of elements of the carrier of $H, I$ be a finite sequence of elements of $\mathbb{Z}$, and $f$ be a homomorphism from $G$ to $H$. Suppose for every element $k$ of $\mathbb{N}$ such that $k \in \operatorname{Seg}$ len $F_{1}$ holds $F_{2}(k)=$ $f\left(F_{1}(k)\right)$ and len $F_{1}=$ len $I$ and len $F_{2}=$ len $I$. Then $f\left(\prod\left(F_{1}{ }^{I}\right)\right)=\prod\left(F_{2}{ }^{I}\right)$.

\section{REFERENCES}

[1] Grzegorz Bancerek. Cardinal numbers. Formalized Mathematics, 1(2):377-382, 1990.

[2] Grzegorz Bancerek. The fundamental properties of natural numbers. Formalized Mathematics, 1(1):41-46, 1990.

[3] Grzegorz Bancerek. The ordinal numbers. Formalized Mathematics, 1(1):91-96, 1990.

[4] Grzegorz Bancerek and Krzysztof Hryniewiecki. Segments of natural numbers and finite sequences. Formalized Mathematics, 1(1):107-114, 1990.

[5] Nicolas Bourbaki. Elements of Mathematics. Algebra I. Chapters 1-3. Springer-Verlag, Berlin, Heidelberg, New York, London, Paris, Tokyo, 1989.

[6] Czesław Byliński. Finite sequences and tuples of elements of a non-empty sets. Formalized Mathematics, 1(3):529-536, 1990.

[7] Czesław Byliński. Functions and their basic properties. Formalized Mathematics, 1(1):5565, 1990.

[8] Czesław Byliński. Functions from a set to a set. Formalized Mathematics, 1(1):153-164, 1990.

[9] Czesław Byliński. Partial functions. Formalized Mathematics, 1(2):357-367, 1990.

[10] Czesław Byliński. Some basic properties of sets. Formalized Mathematics, 1(1):47-53, 1990.

[11] Czesław Byliński. Some properties of restrictions of finite sequences. Formalized Mathematics, 5(2):241-245, 1996.

[12] Agata Darmochwał. Finite sets. Formalized Mathematics, 1(1):165-167, 1990.

[13] Katarzyna Jankowska. Transpose matrices and groups of permutations. Formalized Mathematics, 2(5):711-717, 1991.

[14] Jarosław Kotowicz. Properties of real functions. Formalized Mathematics, 1(4):781-786, 1990.

[15] Beata Padlewska. Families of sets. Formalized Mathematics, 1(1):147-152, 1990.

[16] Andrzej Trybulec. Subsets of complex numbers. To appear in Formalized Mathematics.

[17] Andrzej Trybulec. Tarski Grothendieck set theory. Formalized Mathematics, 1(1):9-11, 1990.

[18] Michał J. Trybulec. Integers. Formalized Mathematics, 1(3):501-505, 1990.

[19] Wojciech A. Trybulec. Classes of conjugation. Normal subgroups. Formalized Mathematics, 1(5):955-962, 1990.

[20] Wojciech A. Trybulec. Groups. Formalized Mathematics, 1(5):821-827, 1990.

[21] Wojciech A. Trybulec. Non-contiguous substrings and one-to-one finite sequences. Formalized Mathematics, 1(3):569-573, 1990.

[22] Wojciech A. Trybulec. Subgroup and cosets of subgroups. Formalized Mathematics, 1(5):855-864, 1990.

[23] Wojciech A. Trybulec. Lattice of subgroups of a group. Frattini subgroup. Formalized Mathematics, 2(1):41-47, 1991.

[24] Wojciech A. Trybulec and Michał J. Trybulec. Homomorphisms and isomorphisms of groups. Quotient group. Formalized Mathematics, 2(4):573-578, 1991.

[25] Zinaida Trybulec. Properties of subsets. Formalized Mathematics, 1(1):67-71, 1990. 
[26] Edmund Woronowicz. Relations and their basic properties. Formalized Mathematics, 1(1):73-83, 1990.

[27] Edmund Woronowicz. Relations defined on sets. Formalized Mathematics, 1(1):181-186, 1990.

Received April 20, 2007 